\title{
Computational pharmacological comparison of Salvia miltiorrhiza and Panax notoginseng used in the therapy of cardiovascular diseases
}

\author{
CHUN-SONG ZHENG ${ }^{1,2}$, XIAO-JIE XU ${ }^{1,3}$, HONG-ZHI YE ${ }^{1,2}$, GUANG-WEN WU ${ }^{2}$, \\ HUI-FENG XU ${ }^{2}$, XI-HAI LI ${ }^{2}$, SU-PING HUANG ${ }^{1}$ and XIAN-XIANG LIU ${ }^{1}$ \\ ${ }^{1}$ Fujian Academy of Integrative Medicine and ${ }^{2}$ Fujian Key Laboratory of Integrative Medicine on Geriatrics, \\ Fujian University of Traditional Chinese Medicine, Fuzhou, Fujian 350122; ${ }^{3}$ College of Chemistry \\ and Molecular Engineering, Peking University, Beijing 100871, P.R. China
}

Received May 6, 2013; Accepted August 30, 2013

DOI: $10.3892 /$ etm.2013.1291

\begin{abstract}
The herb pair comprising Salvia miltiorrhiza (SM) and Panax notoginseng (PN) has been used as a classical formula for cardiovascular diseases (CVDs) in China and in western countries. However, the pharmacology of SM and PN in this herb pair has not been fully elucidated. The aim of this study was to compare the mechanisms of SM and PN at the molecular level for the treatment of CVDs. We used a systems pharmacology approach, integrating ligand clustering, chemical space, docking simulation and network analysis, to investigate these two herbal medicines. The compounds in SM were attached to clusters $2,3,5,6,8$ and 9, while the compounds in PN were attached to clusters $1,2,4,5,6,7,8$ and 10. The distributions of chemical space between the compounds from SM and PN were discrete, with the existence of small portions of overlap, and the majority of the compounds did not violate 'Lipinski's rule of five'. Docking indicated that the average number of targets correlated with each compound in SM and PN were 5.0 and 3.6, respectively. The minority nodes in the SM and PN drug-target networks possessed common values of
\end{abstract}

Correspondence to: Professor Xian-Xiang Liu, Fujian Academy of Integrative Medicine, Fujian University of Traditional Chinese Medicine, 1 Huatuo Road, Minhou Shangjie, Fuzhou, Fujian 350122, P.R. China

E-mail: liuxianxiang@163.com

Abbreviations: TCM, Traditional Chinese Medicine; SM, Salvia miltiorrhiza; PN, Panax notoginseng; CVDs, cardiovascular diseases; TNF- $\alpha$, tumor necrosis factor- $\alpha$; eNOS, endothelial nitric oxide synthase; COX, cyclooxygenase; HO, heme oxygenase; $\operatorname{PPAR} \gamma$, peroxisome proliferator activated receptor $\gamma$; ER, estrogen receptor; HMG-CoA, 3-hydroxy-3-methylglutaryl coenzyme A; ACE, angiotensin-converting enzyme; D-T network, drug-target network

Key words: Salvia miltiorrhiza, Panax notoginseng, cardiovascular disease, computational pharmacology betweenness centrality, closeness centrality, topological coefficients and shortest path length. Furthermore, network analyses revealed that SM and PN exerted different modes of action between compounds and targets. These results suggest that the method of computational pharmacology is able to intuitively trace out the similarities and differences of two herbs and their interaction with targets from the molecular level, and that the combination of two herbs may extend their activities in different potential multidrug combination therapies for CVDs.

\section{Introduction}

Cardiovascular diseases (CVDs) are the leading cause of death globally. Based on data from the World Health Organization (WHO), an estimated 17.3 million people died from CVDs in 2008 , representing $30 \%$ of all global deaths (1). To date, the majority of patients with CVDs have been treated with drug therapies. The main drugs on the market are diuretics, vasodilators, anticoagulants, antiplatelet agents and $\beta$-blockers. While these drugs have yielded desired responses, they have also led to unwanted side-effects $(2,3)$. Multiherb therapy, as an essential component of traditional medicine systems, has been utilized for thousands of years in China and other countries. It has exhibited improved curative efficacies and fewer side-effects, and has been used in the prevention of disease (4). It has been revealed that the majority of herbal medicines exhibit multiple cardiovascular effects (5). Thus, multiherb therapy may be one of the best conventional and complementary medical approaches in the prevention and treatment of CVDs.

Salvia miltiorrhiza (SM) and Panax notoginseng (PN) have been widely used in combination in Traditional Chinese Medicine (TCM) for the therapy of CVDs in China and other countries, including the United States (6-8). It has been demonstrated that these two herbs are compatible and have a synergistic effect (7). However, the molecular mechanisms underlying their compatibility have yet to be clearly elucidated. Numerous computational pharmacological studies, which have been generated using library analysis, quantitative structure-activity relationship (QSAR), receptor-ligand inter- 
action and biological networks, have been developed to clarify the pharmacology and efficacy of TCM $(9,10)$. Therefore, in the present study, we compared the computational pharmacology of SM and PN at the molecular level, in order to enhance the understanding of factors affecting compatibility in TCM and to accelerate modern TCM development.

\section{Materials and methods}

Preparation of SM and PN chemical databases. The structures identified in the medicinal herbs of SM and PN were taken from the Chinese Herbal Drug Database and the Handbook of the Constituents in Chinese Herb Original Plants $(11,12)$. The total number of compounds in SM and PN was 53 and 57, respectively. These compounds were converted into three-dimensional structures and energy optimizations were performed using the Discovery Studio 2.0 (DS 2.0) software (Accelrys Inc., San Diego, CA, USA), based on the Merck Molecular Force Field (MMFF). Following this, the protocol of Cluster Ligands was used to cluster the compounds from the SM and PN chemical databases (13).

Calculation of molecular descriptors. The protocol from 'Calculate Molecular Properties' in the QSAR module of DS 2.0 was employed to calculate the descriptors for the compounds from the SM and PN chemical databases. The chemical space was constructed using 150 diversity descriptors, including the molecular properties of one, two and three dimensions $(14,15)$. Principal component analysis (PCA) was then performed to map the distribution of the compounds in chemical space.

Molecular docking. The modern docking program LigandFit, within DS 2.0, was used to perform the molecular docking. The crystal structures of 16 key proteins associated with CVDs $(16,17)$ were downloaded from the Research Collaboratory for Structural Bioinformatics (RCSB) protein data bank (PDB; Table I; www.rcsb.org). All crystallographic water was removed from the file and hydrogen atoms were added. The inhibitor from the PDB file was used to define the active site. The compounds from the SM and PN chemical databases were docked into the protein models. All docked structures were sorted according to their DockScore. The compounds with the top-five DockScores were selected as potential active compounds, as described previously (18).

Network construction and analysis. Cytoscape 2.8.3 was used for network construction (19). The potential active compounds and their corresponding target proteins were connected to each other to generate a drug-target (D-T) network. In this network, the nodes represented compounds or proteins and the edges represented the compound-target interactions. All data were analyzed using Cytoscape plugins.

\section{Results}

Comparison of the SM and PN chemical databases: Clustering distribution. The compounds from the SM and PN chemical databases were clustered by employing the default settings of Cluster Ligands (Fig. 1). Fig. 1 shows that the compounds in
Table I. Sixteen proteins associated with CVDs.

\begin{tabular}{lcll}
\hline Protein & PDB code & Protein & PDB code \\
\hline TNF- $\alpha$ & 2 AZ5 & Factor IXa & 1X7A \\
eNOS & $1 \mathrm{M} 9 \mathrm{~J}$ & Factor Xa & 1FJS \\
COX-1 & $1 \mathrm{CQE}$ & Factor VIIa & 1 YGC \\
COX-2 & $6 \mathrm{COX}$ & Factor XI & 1 ZSL \\
PPAR $\gamma$ & $2 \mathrm{HFP}$ & HMG-CoA & $1 \mathrm{HW} 8$ \\
HO-1 & $3 \mathrm{TGM}$ & ACE I & $1 \mathrm{UZE}$ \\
Thrombin & 1 YPJ & ACE II & 1 R4L \\
ER & 1 X7J & Renin & $1 \mathrm{BIL}$ \\
\hline
\end{tabular}

CVDs, cardiovascular diseases; PDB, protein data bank; TNF- $\alpha$, tumor necrosis factor- $\alpha$; eNOS, endothelial nitric oxide synthase; COX, cyclooxygenase; PPAR $\gamma$, peroxisome proliferator activated receptor $\gamma$; HO, heme oxygenase; ER, estrogen receptor; HMG-CoA, 3-hydroxy-3-methylglutaryl coenzyme A; ACE, angiotensinconverting enzyme.

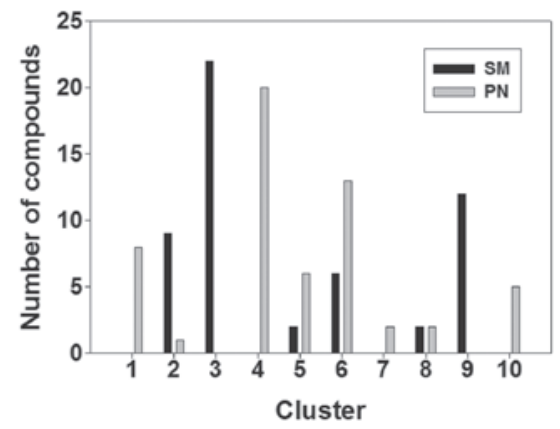

Figure 1. Clustering distribution of compounds from the chemical databases of Salvia miltiorrhiza (SM) and Panax notoginseng (PN).

SM were attached to six clusters, known as clusters 2, 3, 5, 6, 8 and 9, while the compounds in PN were attached to eight clusters, known as clusters $1,2,4,5,6,7,8$ and 10 . These results indicate that SM and PN have similarities and differences with regard to chemical structure clustering.

Comparison of the SM and PN chemical databases: Chemical space. Diversity descriptors $(\mathrm{n}=150)$ were used to map the chemical space of the SM and PN chemical databases using PCA (Fig. 2). A number of the key molecular descriptors of the compounds from the two databases are shown in Table II. The results were as follows: i) Considerable dispersion was observed in the first three principal components; ii) there was only a small overlap between the databases of the two molecules in chemical space; iii) the majority of the molecules did not violate 'Lipinski's rule of five' (20).

Comparison of the SM and PN chemical databases: Compounds with potential biological activity. To investigate whether the compounds in SM and PN were likely to be active in CVDs, the compounds with potential biological activity were predicted using virtual docking. The docking showed that various bioactive compounds in SM and PN targeted multiple proteins associated with CVDs. The average number 
Table II. Maximum, minimum and mean of the molecular descriptors of the SM and PN chemical databases.

\begin{tabular}{|c|c|c|c|c|c|c|}
\hline \multirow[b]{2}{*}{ Descriptors } & \multicolumn{3}{|c|}{ SM } & \multicolumn{3}{|c|}{ PN } \\
\hline & Maximum & Minimum & Mean & Maximum & Minimum & Mean \\
\hline Molecular weight & 718.61 & 154.12 & 326.40 & 1271.44 & 118.18 & 355.78 \\
\hline No. of hydrogen acceptors & 16 & 1 & 4.08 & 28 & 0 & 4.04 \\
\hline No. of hydrogen donors & 9 & 0 & 1.70 & 18 & 0 & 2.49 \\
\hline $\mathrm{A} \log \mathrm{P}$ & 8.08 & 0.61 & 3.57 & 10.41 & -4.54 & 4.34 \\
\hline No. of rotatable bonds & 14 & 0 & 1.98 & 19 & 0 & 7.96 \\
\hline Molecular volume & 438.35 & 88.49 & 219.33 & 853.72 & 86.43 & 270.14 \\
\hline Molecular surface area & 652.14 & 148.91 & 313.64 & 1221.90 & 128.43 & 378.57 \\
\hline Molecular polar surface area & 278.03 & 20.23 & 75.31 & 456.44 & 0 & 67.57 \\
\hline
\end{tabular}

SM, Salvia miltiorrhiza; PM, Panax notoginseng.

Table III. Network properties of the SM and PN D-T networks.

\begin{tabular}{lcc}
\hline Parameters & $\begin{array}{l}\text { SM D-T } \\
\text { network }\end{array}$ & $\begin{array}{r}\text { PN D-T } \\
\text { network }\end{array}$ \\
\hline Network density & 0.161 & 0.114 \\
Network heterogeneity & 0.596 & 0.652 \\
Network centralization & 0.275 & 0.308 \\
Characteristic path length & 3.544 & 2.762 \\
Average no. of neighbors & 5.000 & 4.211 \\
Shortest paths & $992(100 \%)$ & $1,406(100 \%)$ \\
\hline
\end{tabular}

SM, Salvia miltiorrhiza; D-T, drug-target; PN, Panax notoginseng.

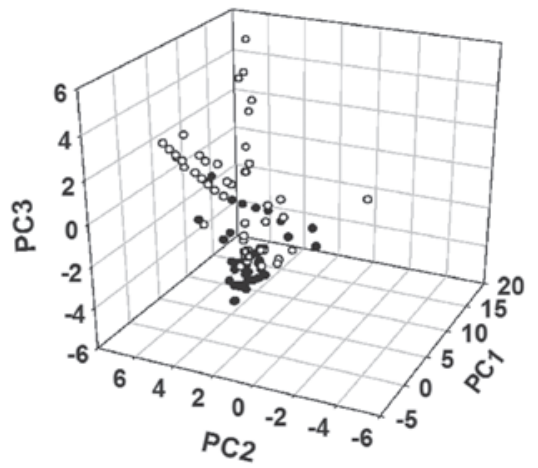

Figure 2. Principal component analysis of compounds from the chemical databases of Salvia miltiorrhiza (SM) and Panax notoginseng (PN). The black and white circles represent the former and the latter, respectively. PC1, first principal component; PC2, second principal component; PC3, third principal component.

of targets correlated with each compound in SM and PN were 5.0 and 3.6 , respectively.

Comparison of the SM and PN chemical databases: D-T network. The D-T network was generated by connecting the potential active compounds to their CVD-associated targets, in order to further clarify the associations between the poten-

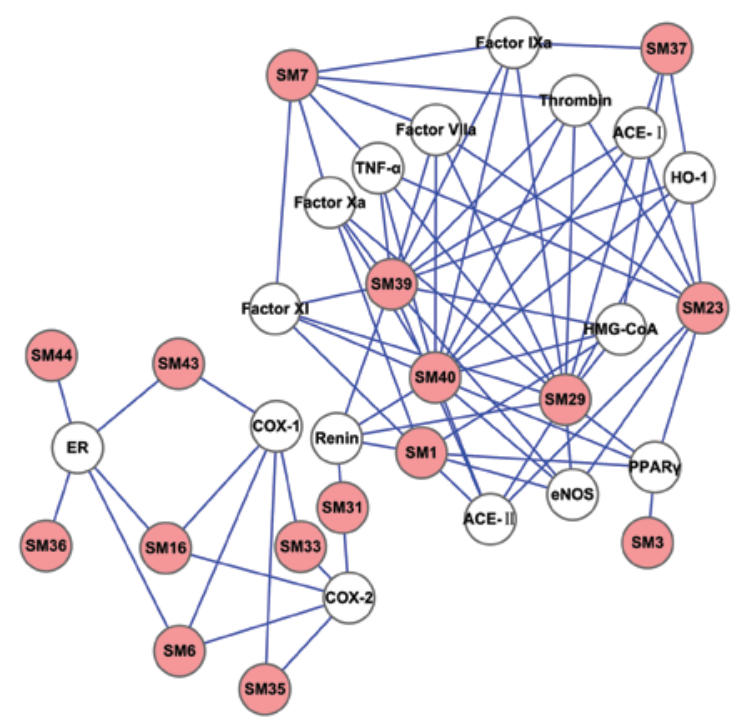

Figure 3. Salvia miltiorrhiza (SM) drug-target network. The white and pink circles represent target proteins associated with cardiovascular diseases (CVDs) and SM compounds, respectively.

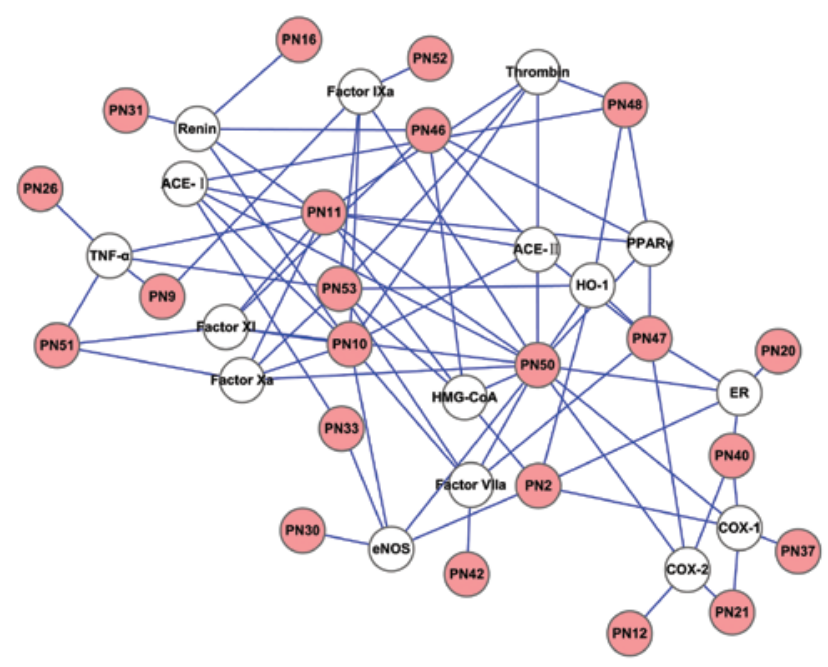

Figure 4. Panax notoginseng (PN) drug-target network. The white and pink represent target proteins associated with cardiovascular diseases (CVDs) and PN compounds, respectively. 
Table IV. Key compounds with the top-five degrees in the SM D-T network and PN D-T network.

\begin{tabular}{|c|c|c|c|c|c|}
\hline \multicolumn{3}{|c|}{ SM D-T network } & \multicolumn{3}{|c|}{ PN D-T network } \\
\hline Index & Chemical name & Degree & Index & Chemical name & Degree \\
\hline SM29 & Monomethyl lithospermate & 13 & PN50 & Quercetin & 15 \\
\hline SM40 & Salvianolic acid C & 13 & PN10 & Dicapryl phthalate & 9 \\
\hline SM39 & Salvianolic acid A & 12 & PN11 & Diisocapryl phthalate & 8 \\
\hline SM23 & Methyl rosmarinate & 8 & PN53 & Stigmasterol & 7 \\
\hline SM1 & Baicalin & 7 & PN47 & Panaxynol & 6 \\
\hline
\end{tabular}

SM, Salvia miltiorrhiza; D-T, drug-target; PN, Panax notoginseng.

A

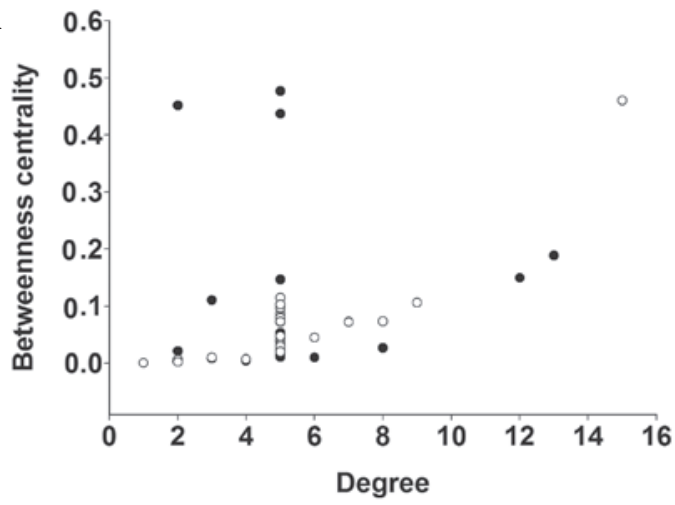

C

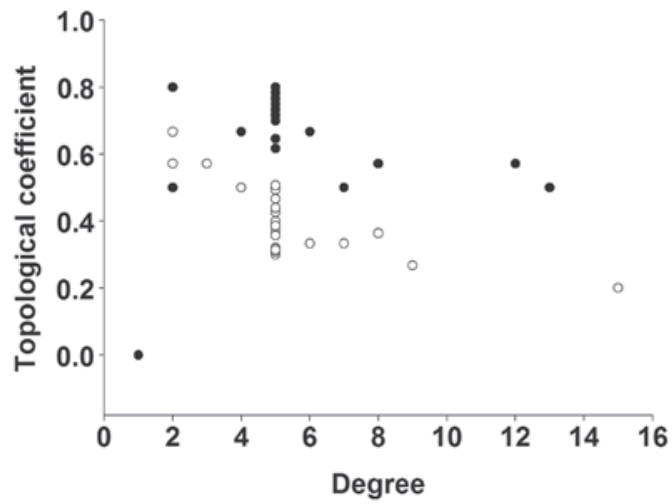

B

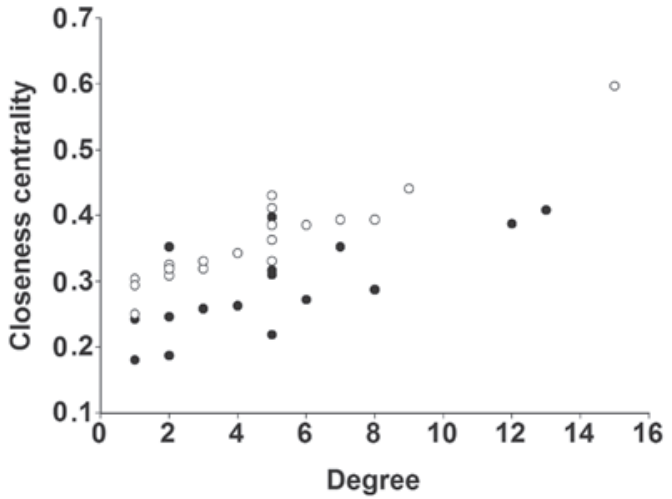

D

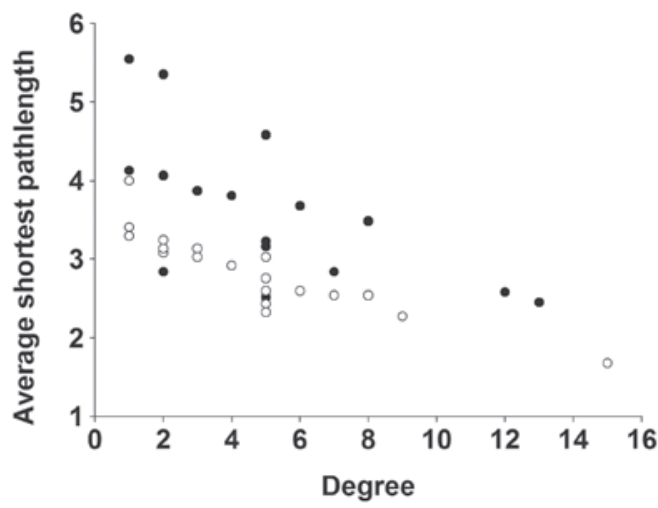

Figure 5. (A-D) Network analyses of Salvia miltiorrhiza (SM) and Panax notoginseng (PN) drug-target (D-T) networks. Parameter statistics of the SM D-T network are shown in black and the parameter statistics of the PN D-T network are shown in white.

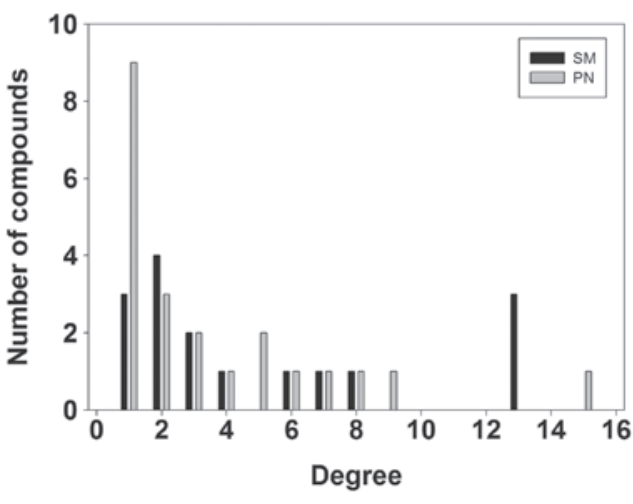

Figure 6. Distribution of the number of targets associated with each compound in Salvia miltiorrhiza (SM) and Panax notoginseng (PN). tially active compounds and their targets (Figs. 3 and 4). The simple parameters of the networks for SM and PN are shown in Table III. Plot parameters of these networks were used to characterize the global map of D-T interactions (Fig. 5) and the degree of compound nodes in the network was analyzed (Fig. 6). The key compounds with the top-five degrees in the two networks are shown in Table IV. These results demonstrated that SM and PN are able to act on multiple targets and exhibit different modes of action between compounds and targets.

\section{Discussion}

CVDs are the leading cause of mortality in the world and pose a serious threat to human health. The diseases are complex and 
multifactorial and are caused by environmental, genetic and clinical risk factors (21). Therefore, CVDs tend to result from multiple molecular abnormalities. As a result, one drug acting on a single target may not lead to the effective treatment of the CVDs $(22,23)$, and drug therapies addressing multiple targets have been receiving increasing focus.

For many years, numerous preparations of the herb pair comprising SM and PN have been used in the treatment of patients with CVDs (24). In the current study, a number of compounds in SM and PN, distributed among the different class groups, are shown in Fig. 1. The results reflect the structural diversity in the molecular composition of SM and PN and the differences between them. SM and PN were shown to possess a broad and different diversity in chemical space (Fig. 2), which indicates that they are likely to exert different effects (15). The statistics of the drug-like properties of the compounds from the SM and PN chemical databases (Table II) revealed the mean molecular weights to be 326.40 and 355.78 , respectively; the mean number of hydrogen bond acceptors was 4.08 and 4.04, respectively; the mean number of hydrogen bond donors was 1.70 and 2.49 , respectively and the mean AlogP was 3.57 and 4.34, respectively. According to the 'rule of five' (20), these compounds have desirable drug-like properties that make them suitable for use as oral drugs in humans. These results provided a good foundation for the screening of suitable active compounds.

A docking screening protocol was used to identify the compounds with multitarget potential for targets associated with CVDs using LigandFit. The docking results showed that the compounds in SM and PN exhibited potential biological activity with one or more target proteins. Among these compounds, $81.25 \%$ of the compounds in SM and $59.05 \%$ of the compounds in PN were able to act on more than one target protein. To further compare the effects of SM and PN on CVDs, we used the screening compounds and their interaction targets to generate a bipartite graph of drug-target interactions, in which a compound and a protein were connected to each other if the protein was an action target of the compound, giving rise to SM (Fig. 3) and PN (Fig. 4) D-T networks. The analyses of these networks (Fig. 5) showed minority common values of betweenness centrality, closeness centrality, topological coefficients and shortest path length, in addition to discriminating the detailed actions of SM and PN on CVDs. Zhu et al (25) proposed that identifying the common behavioral features from the network was likely to provide important information to enable the understanding of the drug-target interaction mechanisms in the human body; therefore, the topological analysis may reflect global knowledge concerning the particular properties of compounds and proteins involved in the network. The results indicated that SM and PN exhibited different modes of action. In addition, the compounds in SM and PN possessed different degrees in the model of the D-T network (Fig. 6 and Table IV). The majority of the compounds in Table IV have been described in previous studies (26-29). As mentioned previously, the herb pair consisting of SM and $\mathrm{PN}$ may possess a range of functions in the treatment of CVDs, via different compounds combining with different targets. Furthermore, the different modes of action of the pair may result in SM and PN exerting synergistic effects in CVDs. Zheng et al (7) demonstrated that SM primarily acted to expand blood vessels, while PN mainly participated in the protection of cardiac myocytes. Therefore, the combination of these two herbs improves coronary circulation and reduces the symptoms of myocardial ischemia. This may further corroborate the proposal that $\mathrm{SM}$ and $\mathrm{PN}$ are able to treat CVDs via different modes of action and that the combination of the two herbs is able to enhance the therapeutic effects.

In conclusion, the results of the present study demonstrated that: i) The compounds in SM and PN have diverse and drug-like properties; ii) the compounds in SM and PN have multitarget potential for targets associated with CVDs, and iii) the combination of SM and PN may enhance their activities in different potential multidrug combination therapies for CVDs. Furthermore, the method of computational pharmacology is able to intuitively trace out the different details of the structural classification, chemical space and modes of action of the compounds in SM and PN. This may be used as a new method for the identification of SM and PN, and to enable the improved understanding of herb pairs at the molecular level.

\section{Acknowledgements}

This study was supported by the Developmental Fund of Chen Keji Integrative Medicine (CKJ2010032).

\section{References}

1. Alwan A, Armstrong T, Bettcher D, Branca F, Chisholm D, Ezzati M, Garfield R, MacLean D, Mathers C, Mendis S, Poznyak V, Riley L, Tang KC and Wild C: Global status report on noncommunicable diseases 2010. WHO Library Cataloguingin-Publication Data, Geneva, pp100-143, 2011.

2. Li SB: Adverse drug reaction and prevention of cardiovascular drugs. Hainan Med J 22(3): 14-19, 2011 (In Chinese).

3. Toyoshima H, Takahashi K and Akera T: The impact of side effects on hypertension management: a Japanese survey. Clin Ther 19: 1458-1469, 1997.

4. Wang S, Hu Y, Tan W, Wu X, Chen R, Cao J, Chen M and Wang Y: Compatibility art of traditional Chinese medicine: from the perspective of herb pairs. J Ethnopharmacol 143: 412-423, 2012.

5. Frishman WH, Sinatra ST and Moizuddin M: The use of herbs for treating cardiovascular disease. Semin Integr Med 2: 23-35, 2004.

6. Liang CZ: Focus on the theory of precaution of disease in Tradition Chinese Medicine from the function of compound Danshen dripping pill to the heart and blood vessel's illness. Chin Arch Trad Chin Med 26: 643-644, 2008 (In Chinese).

7. Zheng Q, Peng CC, Shen ML and Yang M: Study on compatibility of Radix et Rhizoma Salviae miltiorrhizae and Radix et Rhizoma notoginseng. Chin J Exp Trad Med Formulae 15(2): 83-86, 2009 (In Chinese).

8. Cheng TO: Cardiovascular effects of Danshen. Int J Cardiol 121: 9-22, 2007.

9. Zheng CS, Xu XJ, Liu XX and Ye HZ: Computational pharmacology of Jingzhi Tougu Xiaotong granule in preventing and treating osteoarthritis. Acta Phys Chim Sin 26: 775-783, 2010 (In Chinese).

10. Ma S, Feng C, Zhang X, Dai G, Li C, Cheng X, Liu P, Ju W and $\mathrm{Yu} \mathrm{H}$ : The multi-target capabilities of the compounds in a TCM used to treat sepsis and their in silico pharmacology. Complement Ther Med 21: 35-41, 2013.

11. Qiao X, Hou T, Zhang W, Guo S and Xu X: A 3D structure database of components from Chinese traditional medicinal herbs. J Chem Inf Comput Sci 42: 481-489, 2002.

12. Zhou JX, Xie GR and Yang XD: Handbook of the Constituents in Chinese Herb Original Plants. 1st edition. Chemical Industry Press, Beijing, pp1165-1211, 2004.

13. Hassan M, and Bielawski JP, Hempel JC and Waldman M: Optimization and visualization of molecular diversity and combinatorial libraries. Mol Divers 2: 64-74, 1996. 
14. Zheng CS, Xu XJ, Ye HZ, Wu GW, Li XH, Huang SP and Liu XX: Computational approaches for exploring the potential synergy and polypharmacology of Duhuo Jisheng Decoction in the therapy of osteoarthritis. Mol Med Rep 7: 1812-1818, 2013.

15. Dobson CM: Chemical space and biology. Nature 432: 824-828, 2004.

16. Wu DH and Xu XJ: Computational simulation of benefiting Qi and activating blood mechanism of Traditional Chinese Medicine. Acta Phys Chim Sin 25: 446-450, 2009 (In Chinese).

17. Gu JY, Yuan G, Zhu YH and Xu XJ: Computational pharmacological studies on cardiovascular disease by Qishen Yiqi Diwan. Sci China B 52: 1871-1878, 2009.

18. Zheng CS, Ye HZ, Cai LL, Chen JS, Wei LS and Liu XX: Discussion on multi-component and multi-target pattern of Liuwei Dihuang pill in the treatment of osteoarthritis on the basis of computer simulation. J Trad Chin Orthop Traumatol 25: 11-13, 18, 2013 (In Chinese).

19. Smoot ME, Ono K, Ruscheinski J, Wang PL and Ideker T: Cytoscape 2.8: new features for data integration and network visualization. Bioinformatics 27: 431-432, 2011.

20. Lipinski CA, Lombardo F, Dominy BW and Feeney PJ Experimental and computational approaches to estimate solubility and permeability in drug discovery and development settings. Adv Drug Deliv Rev 46: 3-26, 2001.

21. Cooney MT, Dudina A, D'Agostino R and Graham IM: Cardiovascular risk-estimation systems in primary prevention: do they differ? Do they make a difference? Can we see the future? Circulation 122: 300-310, 2010.
22. Baur JA and Sinclair DA: Therapeutic potential of resveratrol: the in vivo evidence. Nat Rev Drug Discov 5: 493-506, 2006.

23. Hopkins AL: Network pharmacology: the next paradigm in drug discovery. Nat Chem Biol 4: 682-690, 2008.

24. Chu Y, Zhang L, Wang XY, Guo JH, Guo ZX and Ma XH: The effect of Compound Danshen Dripping Pills, a Chinese herb medicine, on the pharmacokinetics and pharmacodynamics of warfarin in rats. J Ethnopharmacol 137: 1457-1461, 2011.

25. Zhu M, Gao L, Li X, Liu Z, Xu C, Yan Y, Walker E, Jiang W, Su B, Chen X and Lin H: The analysis of the drug-targets based on the topological properties in the human protein-protein interaction network. J Drug Target 17: 524-532, 2009.

26. Perez-Vizcaino F and Duarte J: Flavonols and cardiovascular disease. Mol Aspects Med 31: 478-494, 2010.

27. Ho JH and Hong CY: Salvianolic acid: small compounds with multiple mechanisms for cardiovascular protection. J Biomed Sci 18: 30, 2011.

28. Ji XF and Shi DH: Advances in studies of baicalin on cardiovascular and cerebrovascular pharmacology. Strait Pharm J 18: 10-12, 2006 (In Chinese).

29. Jiang LP, Nie BM, Lu HM, Gan LJ, Chen HZ and Lu Y: Inhibitory effect of panaxynol on the proliferation of rat aortic smooth muscle cell and its mechanisms. Chin Pharmacol Bull 21: 1313-1319, 2005 (In Chinese). 